\title{
Allgemeinverbindlicherklärung von Tarifverträgen - vom Niedergang zur Reform?
}

\author{
Die nachhaltige Erosion des deutschen Tarifsystems hat zu einer intensiven Diskussion \\ über Möglichkeiten und Wege seiner Restabilisierung geführt. Dabei wird zunehmend \\ die Frage diskutiert, inwieweit durch staatlich-gesetzliche Maßnahmen die Regulierung \\ der Einkommens- und Arbeitsbedingungen auf Basis der Flächentarifverträge gefördert \\ werden kann. Das Instrument der Allgemeinverbindlicherklärung von Tarifverträgen \\ spielt dabei eine wichtige Rolle. Dieser Beitrag analysiert die Entwicklung und Funktion \\ der Allgemeinverbindlicherklärung, ihren Bedeutungsverlust in den vergangenen 20 Jah- \\ ren sowie die darauf bezogene Reformdebatte der jüngsten Zeit.
}

REINHARD BISPINCK

\section{Einleitung und Fragestellung}

Das deutsche Tarifsystem befindet sich seit nunmehr 20 Jahren in einem schleichenden Erosionsprozess, der trotz vielfältiger Aktivitäten insbesondere seitens der Gewerkschaften noch nicht zum Stillstand gekommen ist (Bahnmüller/ Bispinck 1995; Artus 2001; Bispinck 2003; Bahnmüller 2010). Zwar gilt nach wie vor, dass die Mehrzahl der Beschäftigten im Geltungsbereich von Tarifverträgen arbeitet und der branchenbezogene Flächentarifvertrag auch heute noch der dominierende Regelungstyp ist, aber die Prägekraft des Tarifvertrages hat doch in erheblichem Umfang nachgelassen (Abbildung 1). Aktuell gilt, dass inzwischen über $40 \%$ der Beschäftigten und nahezu $70 \%$ der Betriebe nicht mehr einer Tarifbindung unterliegen (Ellguth/Kohaut 2012). Manche Branchen werden inzwischen als weitgehend „tariffreie Zonen“ angesehen. Zu den Problemzonen gehören insbesondere Teile des privaten Dienstleistungsbereichs, die Kleinund Mittelbetriebe und regional betrachtet vor allem Ostdeutschland. Hier beläuft sich die Tarifbindung durch Flächentarifverträge teilweise nur noch auf 10 bis $30 \%$ der Beschäftigten. Die abnehmende Schutzfunktion des Tarifsystems ist auch deswegen problematisch, weil in Deutschland die Beschäftigten umso eher ohne Tarifbindung arbeiten, je geringer ihr Einkommen ist - und das, obwohl gerade im unteren Einkommensbereich die soziale Schutzfunktion des Tarifvertrags von besonderer Bedeutung ist. ${ }^{\circ}$
In einer Situation erodierender Tarifbindung können rechtliche Instrumente zur Ausweitung der Tarifnormen auf nicht tarifgebundene Betriebe („Außenseiter“) eine wichtige stabilisierende Funktion erfüllen. Tatsächlich sieht ja das Tarifvertragsgesetz (TVG) seit $1949 \mathrm{im} \$ 5$ eine solche Möglichkeit vor, von der in der Praxis vielfältig Gebrauch gemacht wurde. Die mittlerweile aber stark rückläufige Zahl der Allgemeinverbindlicherklärungen (AVE) - nicht nur, aber ganz besonders im Bereich der Lohn- und GehaltsTarifverträge - führt zu der These vom Niedergang dieses Instruments, der im Folgenden nachgegangen werden soll. Ihr widerspricht auf den ersten Blick eine gegenläufige Entwicklung im Bereich des Arbeitnehmer-Entsendegesetzes (AEntG). Die dort vorgesehene Möglichkeit der Festlegung von Mindestlöhnen auf Basis einer Allgemeinverbindlicherklärung bzw. einer Rechtsverordnung ist in den vergangenen Jahren deutlich stärker genutzt worden.

Als Reaktion auf die offenkundig dauerhaft nachlassende Tarifbindung und den schleichenden Funktionsverlust der Allgemeinverbindlicherklärung entwickelte sich in den vergangenen Jahren eine zunächst eher verhaltene

Nach den Daten der WSI-Lohnspiegel-Datenbank beträgt die Tarifbindung im untersten Einkommensquintil $32 \%$ und steigt dann gleichmäßig an bis auf $68 \%$ im obersten Quintil. Dies weicht deutlich von der Situation in anderen europäischen Ländern ab (Tijdens/van Klaaveren 2009). 
Diskussion um mögliche Maßnahmen zur aktiven politischen Stützung des Tarifsystems. Sie führte in jüngster Zeit auch zu Initiativen der politischen Parteien, die sich explizit auf die Reform des Instruments der AVE bezogen. Der folgende Beitrag fragt daher auch nach den regulierungspolitischen Konzepten und Zielvorstellungen der Reformvorschläge. ${ }^{2}$

\section{Grundlagen der Allgemeinver- bindlicherklärung}

\subsection{Von der Tarifverordnung zum Tarifvertragsgesetz}

Das Instrument der Allgemeinverbindlicherklärung (AVE) reicht bis in die Anfänge der Weimarer Republik zurück (Wiedemann 2007, S. 9ff; Kempen/Zachert 2006, S. 1078ff.). Mit der Novemberrevolution erfolgte „ein Durchbruch hinsichtlich der rechtlichen Erfassung und Normierung des Tarifvertrages" (Kempen/Zachert 2006, S. 65), die in der „Verordnung über Tarifverträge, Arbeiter- und Angestelltenausschüsse und Schlichtung von Arbeitsstreitigkeiten 23.12.1918“ zum Ausdruck kamen. Die Tarifverordnung erkannte die unmittelbare und zwingende Wirkung des Tarifvertrages an und schuf erstmals die Möglichkeit, Tarifverträge für allgemeinverbindlich zu erklären. Der damalige Reichsarbeitsminister konnte eine solche Erklärung vornehmen, wenn die Tarifverträge für die Gestaltung der Arbeitsbedingungen in dem jeweiligen Tarifgebiet eine „überwiegende Bedeutung“ hatten. Voraussetzung war ein Antrag mindestens einer der Tarifparteien. ${ }^{\text {( }}$ Gedacht war das Instrument als Mittel zum Schutz der tarifgebundenen Beschäftigten vor „Schmutzkonkurrenz" durch Beschäftigte mit untertariflicher Bezahlung. Vor dem Hintergrund der krisenhaften Arbeitsmarktentwicklung gewann die AVE rasch an Bedeutung. ${ }^{0}$ In den zehn Jahren des Bestehens der Tarifverordnung wurden mehr als 25.000 AVEs ausgesprochen. Ende 1929 bestand für 1.846 Tarifverträge eine AVE, das entsprach einem Anteil von 20,5\%, bei den Angestelltentarifverträgen waren es 789 Tarifverträge mit einem Anteil von $44,7 \%{ }^{\odot}$ Insgesamt galten für rund die Hälfte aller von Tarifverträgen erfassten Beschäftigten Allgemeinverbindlicherklärungen.

Unter den Nationalsozialisten wurde durch das Gesetz zur Ordnung der nationalen Arbeit von 1934 die Möglichkeit, Tarifverträge abzuschließen, abgeschafft. Damit waren auch Allgemeinverbindlicherklärungen nicht mehr möglich. An die Stelle der Tarifverträge traten die von den „Treuhändern der Arbeit" festgesetzten Tarifordnungen. Bereits in der unmittelbaren Nachkriegszeit wurde das Instrument der AVE erneut diskutiert und in einzelnen Ländern (Baden, Bayern) sogar in den Landesverfassungen abgesichert. Im Tarifvertragsgesetz vom 9.4.1949 wurde die AVE dann ge-
ABB. 1

\section{Tarifbindung durch Branchentarifverträge 1998-2011 nach Beschäftigten und Betrieben}

Angaben in Prozent

- Beschäftigte Westdeutschland

- Beschäftigte Ostdeutschland

- Betriebe Westdeutschland

- - Betriebe Ostdeutschland

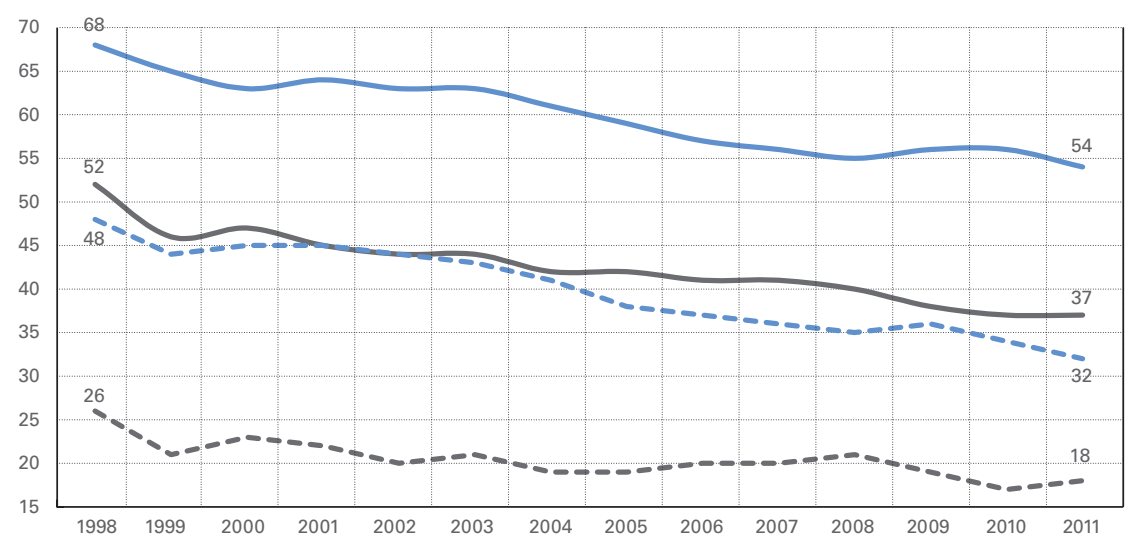

Quelle: Institut für Arbeitsmarkt- und Berufsforschung

setzlich verankert. Allerdings hatte es im Vorfeld Kontroversen über die konkrete Ausgestaltung gegeben. Dabei ging es um ein mögliches Initiativrecht der Arbeitsverwaltung, die Rolle der Länder, die Frage des Antragserfordernisses durch die Tarifparteien und um die inhaltlichen Voraussetzungen einer AVE. ${ }^{\circledR}$ Die Militärverwaltung drängte dabei auf eine restriktive Ausgestaltung der Regelungen. Sie legte Wert auf die Festschreibung eines $50 \%$-Quorums und lehnte die weitere vorgesehene Begründung „Erfordernis der sozialen Ordnung“ ab (Nautz 1985, S. 131ff.; Wonneberger 1992, S. 7ff.).

(2) Siehe dazu auch die Beiträge von Däubler und Rödl in diesem Heft.

(3) Die Anträge wurden bekannt gemacht, in einem bestimmten Zeitraum konnten Einwendungen erhoben werden, die Tarifvertragsparteien wurden zu "Äußerungen" aufgefordert, anschließend entschied der Reichsarbeitsminister auf dieser Basis endgültig. Die allgemeinverbindlichen Tarifverträge wurden in das Tarifregister eingetragen.

(4) Die folgenden Angaben nach Wank, in: Wiedemann 2007, S. 1516.

(5) Goldschmidt (1950) berichtet für Ende Dezember 1930 eine Zahl von 2.062 allgemeinverbindlichen Tarifverträgen.

6 Die Gewerkschaften legten Wert darauf, dass eine AVE lediglich auf Antrag derTarifparteien zustande kommen könne. In Vorentwürfen war sogar eine Verpflichtung zur AVE vorgesehen, sofern beide Tarifparteien dies wünschten (Wonneberger 1992, S. 8). 
Im Tarifvertragsgesetz von 1949 wurde die Allgemeinverbindlicherklärung im $\$ 5$ dann in folgender Form geregelt:

- Eine AVE muss von (mindestens) einer Tarifvertragspartei beantragt werden. Das Bundesarbeitsministerium kann nicht von Amts wegen tätig werden.

- Die tarifgebundenen Arbeitgeber müssen mindestens $50 \%$ der unter den Geltungsbereich des Tarifvertrags fallenden Arbeitnehmer beschäftigen.

- Die Allgemeinverbindlicherklärung muss im öffentlichen Interesse geboten erscheinen.

- Erst in einer Änderung des Tarifvertragsgesetzes 1952 wurde eingefügt, dass zur Behebung eines sozialen Notstandes ebenfalls eine AVE erteilt werden kann, ohne dass die beiden Kriterien (50 \%-Quorum, öffentliches Interesse) erfüllt sein müssen.

Der Bundesarbeitsminister kann, wenn diese Voraussetzungen vorliegen, einen Tarifvertrag für allgemeinverbindlich erklären. Dazu ist das Einvernehmen des Tarifausschusses erforderlich, der sich aus je drei Vertretern der Spitzenorganisationen der Arbeitgeber und der Arbeitnehmer zusammensetzt. Das bedeutet, dass die Mehrheit der Mitglieder zustimmen muss. Das genaue AVE-Verfahren ist in einer entsprechenden Durchführungsverordnung festgelegt.

\subsection{Allgemeinverbindlicherklärung im Arbeitnehmer-Entsendegesetz}

Das Arbeitnehmer-Entsendegesetz (AEntG) von 1996, das der Umsetzung der entsprechenden EU-Entsenderichtlinie dient, greift ebenfalls auf das Instrument der AVE zurück. Es regelt in $₫ 3$, dass bestimmte für allgemeinverbindlich erklärte Tarifverträge auch auf Arbeitnehmer angewendet werden müssen, die in Deutschland beschäftigt sind, deren Arbeitgeber ihren Firmensitz jedoch im Ausland haben. Der Geltungsbereich des Gesetzes war zu Beginn auf das Baugewerbe mit verschiedenen Branchen beschränkt und wurde bis heute schrittweise auf acht Wirtschaftsbereiche ausgeweitet. Der sachliche Geltungsbereich dieser Tarifverträge ist auf folgende Punkte beschränkt:

- Mindestentgeltsätze einschließlich Überstundensätze,

- Urlaubsdauer, Urlaubsentgelt und zusätzliches Urlaubsgeld,

- Höchstarbeitszeiten und Mindestruhezeiten,

- Bedingungen für die Arbeitnehmerüberlassung,

- Arbeits- und Gesundheitsschutz sowie Schutz besonderer Personengruppen,

- Gleichbehandlung von Männern und Frauen und andere Nichtdiskriminierungsbestimmungen.

Alternativ kann neben einer AVE auch eine Rechtsverordnung nach $\$ 7$ AEntG als Grundlage für die zwingende Anwendung der Tarifverträge dienen. Voraussetzung ist ein Antrag beider Tarifparteien auf Allgemeinverbindlicherklä- rung. Damit ist die Zustimmung der Arbeitgeber im Tarifausschuss nicht mehr zwingende Voraussetzung für die Durchsetzung eines Mindestlohntarifvertrages. ${ }^{0}$

Die Reichweite und Bedeutung des AEntG ist durch seine Neufassung aus dem Jahr 2009 deutlich gestiegen. Zweifelsohne hatte die Reform des AEntG maßgeblich die Funktion einer politischen Entlastungsstrategie in der Auseinandersetzung um einen branchenübergreifenden gesetzlichen Mindestlohn (Bispinck/Schulten 2008).

\subsection{Funktionen der Allgemeinverbindlich- erklärung}

Tarifverträge zielen auf die Aufhebung der Unterbietungskonkurrenz der abhängig Beschäftigten, auf die Schaffung gleicher Einkommens- und Arbeitsbedingungen und insofern auch auf gleiche Wettbewerbsbedingungen für die Unternehmen im Hinblick auf Arbeitskosten und Personaleinsatz. Tarifnormen und -regelungen gelten aber unmittelbar und zwingend nur für die tarifgebundenen Betriebe und Beschäftigten einer Branche. Die AVE stellt das zentrale Instrument dar, diese Wirkung eines Tarifvertrages auf den gesamten Tarifbereich und damit auch auf Außenseiter zu erstrecken. Auf diese Weise sollen wirtschaftlich und sozial unerwünschte Folgen aus der begrenzten Tarifbindung unterbunden werden. Daran haben die Tarifparteien beider Seiten bis zu einem gewissen Grad ein gemeinsames Interesse. Bei genauerer Betrachtung lassen sich folgende Zielsetzungen und Funktionen der AVE unterscheiden: ${ }^{8}$

(1) Schutz tarifvertraglicher Normsetzung vor Schmutzkonkurrenz: Schmutzkonkurrenz liegt vor, wenn nicht tarifgebundene Arbeitgeber sich durch untertarifliche Beschäftigung eine günstigere Personalkostensituation und dadurch einen „sozial unfairen“ Wettbewerbsvorteil verschaffen. Dies erhöht den Druck auf die tarifgebundenen Arbeitgeber, die Tarifbindung womöglich aufzugeben, um nicht Marktanteile zu verlieren oder ganz vom Markt verdrängt zu werden. Neue hinzutretende Arbeitgeber werden im Zweifel von vornherein auf eine Tarifbindung verzichten. Indirekt wächst auch der Druck auf die gewachsenen Tarifstandards, die ggf. bei weiteren Tarifverhandlungen zur Disposition gestellt werden. Mittelfristig besteht die Gefahr

(7) Dieser Regelung war ein scharfer Konflikt um den Tarifvertrag Mindestlohn Bau aus dem Jahr 1996 vorausgegangen. Die AVE war im Tarifausschuss von der Arbeitgeberseite abgelehnt worden. Erst nach monatelangen Auseinandersetzungen, die u. a. zu einer Absenkung des vereinbarten Mindestlohnes um 17 \% führten, erklärte sich die Bundesvereinigung der Deutschen Arbeitgeberverbände (BDA) zu einer auf acht Monate befristeten AVE bereit (Däubler 1997; Dombre 2007).

8 Vgl. zum Folgenden genauer Kirsch/Bispinck 2002; Kirsch 2003; Kreimer-de Fries 1995; Wonneberger 1992. 
der Destabilisierung der Tarifsituation in der betroffenen Branche.

(2) Schaffung sozial angemessener Arbeitsbedingungen für Außenseiter: Die Verhinderung von sozial unfairen Wettbewerbsvorteilen z. B. durch Lohndumping bedeutet zugleich auch die Schaffung sozial angemessener Arbeitsbedingungen für die nicht organisierten Arbeitnehmerinnen und Arbeitnehmer. Dies kann als eigenständige Zweckbestimmung der AVE angesehen werden, unabhängig davon, ob die tarifliche Normsetzung als solche gefährdet ist. Dass die nicht organisierten Beschäftigten auf diese Weise quasi automatisch und mühelos in den Genuss tariflicher Regelungen und Leistungen gelangen, ist den Gewerkschaften sicherlich „ein Dorn im Auge“ und Ursache für eine grundlegende Skepsis mancher Gewerkschaften gegenüber dem Instrument.

(3) Sicherung der Funktionsfähigkeit gemeinsamer Einrichtungen der Tarifparteien: In manchen Branchen bestehen gemeinsame Einrichtungen wie z. B. Sozialkassen (zur Sicherung von Urlaubsansprüchen, zusätzlicher Altersversorgung u. a. m.), die nur bei flächendeckender Teilnahme der Betriebe einer Branche dauerhaft funktionsfähig sind (vgl. den Beitrag von Asshoff in diesem Heft). Wenn diese Einrichtungen lediglich von den tarifgebundenen Arbeitgebern getragen würden, wäre die Finanzierungsbasis im Zweifel nicht ausreichend und es käme zu einer de facto Subventionierung der Außenseiterbetriebe. Die AVE garantiert diese umfassende Einbeziehung auch der Außenseiterbetriebe und verhindert auf diese Weise auch ein „Free-Rider-Verhalten“ der nicht tarifgebundenen Unternehmen.

(4) Umsetzung gesetzgeberischer Vorhaben: In den vergangenen Jahrzehnten hat es immer wieder sozial- und arbeitsmarktpolitische Gesetze gegeben, die in ihrer Umsetzung auf die tarifpolitische Begleitung ausgelegt und angewiesen waren (Bispinck 2012). Zu den Regelungsfeldern zählen die zusätzliche Altersversorgung, die Altersteilzeit, Fragen der beruflichen Aus- und Weiterbildung u. a. m. Der Einsatz der AVE trägt dazu bei, dass solche tariflichen Regelungen einen deutlich größeren Wirkungskreis entfalten und damit auch die sozialpolitische Zielsetzung besser erfüllt wird.

\section{Entwicklung der Allgemeinver- bindlicherklärung}

\subsection{Allgemeine Entwicklung}

Die Nutzung des Instruments der AVE erreichte nach seiner Neueinführung im Jahr 1949 zu keiner Zeit das Ausmaß der Praxis in der Weimarer Republik. Gleichwohl machten die Tarifparteien von Anfang an von der Möglichkeit des
$\S 5$ TVG regen Gebrauch. Nach 43 Anträgen im Jahre 1949, von denen 17 bewilligt wurden, belief sich die Zahl der Anträge in den kommenden zehn Jahren durchschnittlich auf knapp 150, im Laufe der 1960er Jahre ging die Zahl der Anträge deutlich auf knapp 90 zurück, um in den 1970er Jahren auf fast 200 sichtbar anzusteigen (Wonneberger 1992, S. 27).

Einer Bilanz der ersten 20 Jahre des TVG durch das Bundesministerium für Arbeit und Soziales (BMA) lässt sich entnehmen, dass für insgesamt 2.501 Tarifverträge eine AVE beantragt wurde, nur in wenigen Fällen wurde dem Antrag nicht entsprochen (Reichel 1969, S. 370). Das entspricht rein rechnerisch einem Jahresdurchschnitt von 125 Anträgen. Ende 1968 bestanden 158 gültige AVE, die meisten entfielen auf Branchen aus dem Bau- und Baunebengewerbe, gefolgt vom Handel und handelsnahen Bereichen.

Bei der Analyse der AVE-Entwicklung ist im Weiteren zwischen den alten und neuen Bundesländern zu unterscheiden. In den alten Bundesländern lag die Blütezeit des Instruments in quantitativer Hinsicht in der zweiten Hälfte der 1970er und Anfang der 1980er Jahre. Seitdem ging die Zahl der gültigen AVE kontinuierlich zurück, unterbrochen von einer kurzen Steigerung zu Beginn der 1990er Jahre. ${ }^{\circ}$ 1991 betrug die Zahl der allgemeinverbindlichen Tarifverträge 615, im Jahr 2011 nur noch 319. Das entspricht einem Rückgang um 48 \%. Dabei spielten zwei Faktoren eine Rolle: Die Zahl der neu allgemeinverbindlich erklärten Tarifverträge, die Mitte der 1970er Jahre noch bei über $200 \mathrm{im}$ Jahr gelegen hatte, ging seit 1990 von damals 176 nahezu durchgängig zurück und belief sich 2011 nur noch auf zwölf. Zugleich überstieg die Zahl der außer Kraft tretenden AVEs in den meisten Jahren die Zahl der Neuzugänge.

In den neuen Bundesländern stellt sich die Entwicklung anders dar (Abbildung 2): Nach der Übertragung der Tarifstrukturen aus den alten Bundesländern stieg in den ersten zehn Jahren die Zahl der allgemeinverbindlichen Tarifverträge auf 171 (2001) an und verharrt seitdem einigermaßen stabil auf diesem Niveau. Allerdings zeigt sich hier, dass die Dynamik bei den neu allgemeinverbindlich erklärten Tarifverträgen praktisch zum Erliegen gekommen ist: Wurden in den ersten Jahren jährlich zwischen 45 und 55 Verträge neu allgemeinverbindlich erklärt, ging dieser Wert in den letzten Jahren auf unter zehn zurück.

Insgesamt wurde die Genehmigungspraxis im Lauf der Jahre rigider. Lag die Quote der abgelehnten Anträge in den 1990er Jahren bei $6 \%$, lag sie im folgenden Jahrzehnt mit $11 \%$ fast doppelt so hoch. Hinzu kommt, dass eine Reihe von Anträgen auch während des Verfahrens zurückgezogen wurde.

Da in der Gesamtzahl der allgemeinverbindlichen Tarifverträge auch eine größere Zahl von Änderungstarif-

(9 Vgl. für den Zeitraum seit Anfang der 1991-2002 ausführlich Kirsch (2003). 


\section{Allgemeinverbindlicherklärungen in Westdeutschland 1976-2011 und Ostdeutschland 1991-2011}
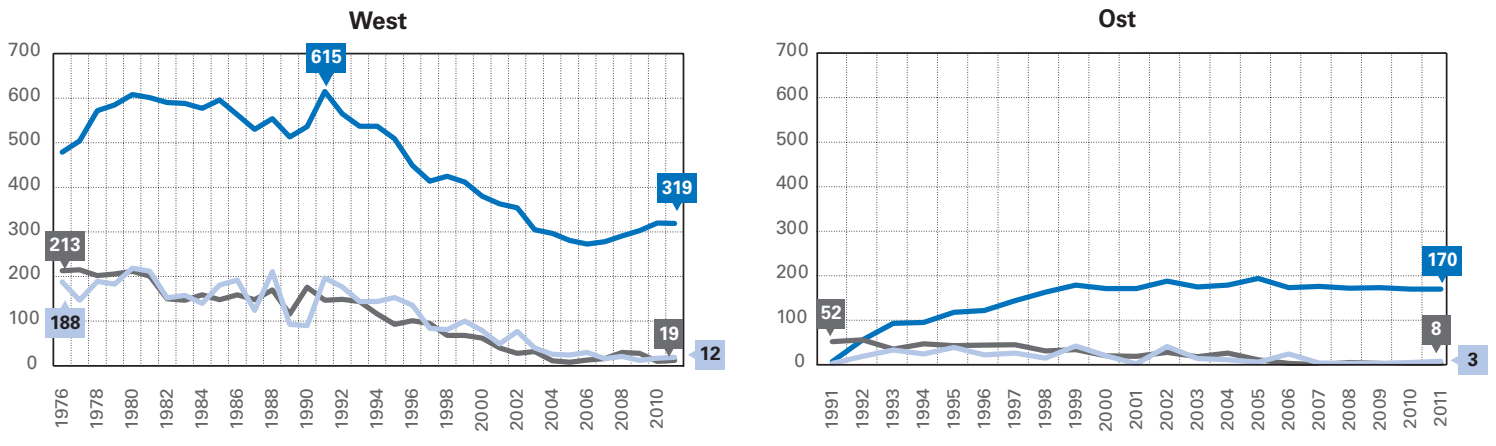

verträgen, Protokollnotizen o. ä. enthalten ist, lohnt ein Blick auf die Entwicklung der Zahl der Ursprungstarifverträge (Tabelle 1). Sie ging im Verlauf der vergangenen zwei Jahrzehnte von 408 (1991) auf 233 (2011) ebenfalls stark zurück. Bezieht man diese Zahl auf die Zahl der Ursprungsverbandstarifverträge insgesamt, erhält man eine Maßzahl, die die praktische Relevanz des Instruments in einer ersten Annäherung zum Ausdruck bringt. Bereits Anfang der 1990er Jahre lag dieser Anteil mit 5,4\% relativ niedrig, insbesondere wenn man dies mit den Verhältnissen in anderen europäischen Ländern vergleicht, die das Instrument intensiver nutzen. ${ }^{\circledR}$ Im Jahr 2011 betrug der Anteil nur noch 1,7 \%.

\subsection{Branchen und Tarifvertragsarten}

Traditionell wird das Instrument der AVE in den einzelnen Wirtschaftsbereichen und den einzelnen tariflichen Regelungsbereichen in sehr unterschiedlichem Umfang genutzt. Dabei zeigt sich im Laufe der Jahrzehnte ein deutlicher Wandel. Für die ersten vier Nachkriegsjahrzehnte zeigt Wonneberger (1992, S. 28ff.), dass $80 \%$ und mehr der allgemeinverbindlichen Ursprungstarifverträge auf sechs Wirtschaftsbereiche entfielen. In den 1950er und 1960er Jahren dominierte der Handel (insbesondere der Großhandel, auf den zeitweise $60 \%$ der AVE entfielen), in den 1970er und 1980er Jahren stellte die Textil- und Bekleidungsindustrie den größten Anteil, und über den gesamten Zeitraum spielte auch das Baugewerbe eine bedeutende Rolle. Weitere relevante Bereiche waren das Handwerk und der Bereich Steine/Baustoffe/Keramik.

Die Daten des BMA-Tarifregisters zeigen auch am aktuellen Rand eine sehr ungleichmäßige Verteilung der allgemeinverbindlichen Tarifverträge nach Branchen. Anfang 2011 waren insgesamt 488 Tarifverträge allgemeinverbindlich. 80 \% entfielen auf nur fünf von 27 Wirtschaftsgruppen.
Allein für das Baugewerbe waren es 202 allgemeinverbindliche Tarifverträge, gefolgt von der Wirtschaftsgruppe Steine, Erden, Keramik, Glas mit 78 Verträgen, der Wirtschaftsgruppe Entsorgung, Reinigung und Körperpflege mit 46 Verträgen, dem Bereich Textil mit 33 sowie dem Bereich Nahrung und Genussmittel mit 28 Verträgen.

Wie war nun die Entwicklung in den vergangenen 20 Jahren? Abbildung 3 zeigt anhand der aussagekräftigeren Zahlen für die allgemeinverbindlichen Ursprungstarifverträge zwar in allen Wirtschaftsgruppen einen deutlichen Rückgang der AVE, in den einzelnen Bereichen fällt die Entwicklungsdynamik jedoch unterschiedlich aus:

- Der Handel startete von einem hohen Niveau und ist seit dem Jahr 2000 geradezu abgestürzt und verharrt seit Mitte des vergangenen Jahrzehnts auf einem sehr niedrigen Niveau.

- Eine ähnliche Entwicklung, von einem etwas niedrigeren Ausgangsniveau aus, zeigt sich für die Bereiche Textil sowie Reinigung und Körperpflege. Im Letztgenannten hat es allerdings seit 2007 einen deutlichen Erholungsprozess gegeben.

- Auch im Baugewerbe zeigte sich nach einer Rückgangsphase seit Mitte der 2000er Jahre ein Wiederanstieg der AVE-Zahlen auf relativ hohem Niveau.

- Kontinuierliche Rückgänge gab es auch in den Bereichen Bekleidung, Nahrung, Genussmittel sowie Eisen, Stahl, Metall, wobei das Ausgangsniveau teils deutlich niedriger lag. - Im Bereich Gaststätten und Beherbergung verlief die Entwicklung relativ stabil, allerdings auf sehr niedrigem Niveau.

(10 Vgl. die Beiträge von Schulten, Rojer/Veldt sowie Dufresne/ Maggi-Germain in diesem Heft. 
Allgemeinverbindliche Tarifverträge und allgemeinverbindliche Ursprungstarifverträge 1991-2011

Angaben in absoluten Zahlen und in Prozent

\begin{tabular}{|c|c|c|c|c|c|c|c|c|}
\hline \multirow[t]{3}{*}{ Jahr } & \multicolumn{5}{|c|}{ Allgemeinverbindliche Tarifverträge insgesamt } & \multicolumn{3}{|c|}{ Allgemeinverbindliche Ursprungstarifverträge } \\
\hline & 1 & 2 & 3 & & & 5 & 6 & 7 \\
\hline & $\begin{array}{l}\text { Bestand } \\
\text { 1. Januar } \\
\text { des Jahres }\end{array}$ & $\begin{array}{c}\text { AVE im Laufe } \\
\text { des Jahres } \\
\text { erklärt }\end{array}$ & $\begin{array}{c}\text { AVE im Laufe } \\
\text { des Jahres } \\
\text { beantragt }\end{array}$ & & $\begin{array}{l}\text { ter: } \\
\text { hnte } \\
\text { äge } \\
\quad \text { in } \%\end{array}$ & $\begin{array}{l}\text { Bestand } \\
\text { 1. Januar } \\
\text { des Jahres }\end{array}$ & $\begin{array}{l}\text { Anteil an allen } \\
\text { allgemeinverbindlichen } \\
\text { Tarifverträgen in \% }\end{array}$ & $\begin{array}{l}\text { Anteil an allen gültigen } \\
\text { Ursprungsverbands } \\
\text { tarifverträgen }{ }^{1} \text { in \% }\end{array}$ \\
\hline 1991 & 621 & 199 & 303 & 18 & 6 & 408 & 66 & 5,4 \\
\hline 1992 & 621 & 205 & 275 & 8 & 3 & 407 & 66 & 5,1 \\
\hline 1993 & 630 & 179 & 257 & 9 & 3,5 & 398 & 63 & 4,8 \\
\hline 1994 & 632 & 163 & 231 & 2 & $<1$ & 402 & 64 & 4,7 \\
\hline 1995 & 627 & 136 & 187 & 10 & 5 & 405 & 65 & 4,5 \\
\hline 1996 & 571 & 145 & 192 & 8 & 4 & 378 & 66 & 4,1 \\
\hline 1997 & 558 & 140 & 191 & 9 & 5 & 333 & 60 & 3,8 \\
\hline 1998 & 588 & 99 & 143 & 17 & 12 & 347 & 59 & 3,5 \\
\hline 1999 & 592 & 102 & 117 & 12 & 10 & 330 & 56 & 3,2 \\
\hline 2000 & 651 & 82 & 136 & 21 & 15 & 298 & 54 & 2,9 \\
\hline 2001 & 534 & 61 & 77 & 9 & 12 & 280 & 52 & 2,6 \\
\hline 2002 & 542 & 70 & 88 & 6 & 6 & 282 & 52 & 2,5 \\
\hline 2003 & 480 & 76 & 102 & 11 & 11 & 262 & 55 & 2,2 \\
\hline 2004 & 476 & 42 & 46 & 1 & 2 & 261 & 55 & 2,0 \\
\hline 2005 & 475 & 31 & 40 & 1 & 3 & 243 & 51 & 1,8 \\
\hline 2006 & 446 & 33 & 44 & 7 & 16 & 230 & 52 & 1,5 \\
\hline 2007 & 454 & 36 & 53 & 7 & 13 & 237 & 52 & 1,5 \\
\hline 2008 & 463 & 29 & 40 & 9 & 23 & 240 & 52 & 1,5 \\
\hline 2009 & 476 & 28 & 48 & 6 & 13 & 244 & 51 & 1,5 \\
\hline 2010 & 490 & 21 & 27 & 3 & 11 & 243 & 50 & 1,5 \\
\hline 2011 & 489 & 13 & 19 & 3 & 16 & 233 & 48 & 1,7 \\
\hline
\end{tabular}

'Allgemeinverbindliche Ursprungstarifverträge jeweils zum Stichtag 1. Januar; Gesamtzahl der Ursprungsverbandstarifverträge sind jeweils zwischen Gewerkschaft und Arbeitgeberverband für einen bestimmten Tarifbereich abgeschlossene originäre Tarifverträge, d. h. keine Änderungstarifverträge, Protokollnotizen o.A. jeweils zum Stichtag 31.12. des Vorjahres.

Quelle: BMA-Tarifregister, Berechnungen des Autors.

Mitteilungen

Betrachtet man die Verteilung der allgemeinverbindlichen Ursprungstarifverträge nach Regelungsbereichen, stellt man fest, dass aktuell (Stand 2011) der größte Teil (61 \%) auf Tarifverträge zu einzelnen Mantelbestimmungen entfällt. ${ }^{\text {(1) }}$ Mit deutlichem Abstand folgen komplette Manteltarifverträge mit $24 \%$, während Einkommenstarifverträge lediglich $15 \%$ der Verträge ausmachen. Im Zeitraum der vergangenen 20 Jahre ist bei allen drei Arten ein deutlicher Rückgang zu verzeichnen. Am stärksten fiel er mit $71 \%$ bei den Einkommenstarifverträgen aus.

\subsection{Allgemeinverbindliche Einkommens- tarifverträge}

Die Daten zeigen, dass die Nutzung des Instruments der AVE bei Einkommenstarifverträgen im Laufe der Jahrzehnte sehr stark zurückgegangen ist. Die Lohn- und Gehaltstarifverträge hatten in den ersten vier Jahrzehnten im AVE-
Geschehen durchweg eine große Bedeutung: Ihr Anteil an den allgemeinverbindlichen Tarifverträgen bewegte sich zwischen 30 und 60 \% (Tabelle 2). Der starke Rückgang in der zweiten Hälfte der 1980er Jahre hängt im Wesentlichen mit dem Wegfall der AVE im Einzelhandel und der Textilund Bekleidungsindustrie zusammen.

Die Zahlen des BMA-Tarifregisters für die vergangenen zwei Jahrzehnte zeigen eine kontinuierliche Fortsetzung dieses Trends bis heute (Tabelle 3). Die Zahl der allgemeinverbindlichen Ursprungstarifverträge zu Lohn und Gehalt ist von $124 \mathrm{im}$ Jahr 1991 auf $36 \mathrm{im}$ vergangenen Jahr

(11) Sie umfassen u. a. Regelungen zu vermögenswirksamen Leistungen, Urlaub/Urlaubsgeld, zusätzlicher Altersversorgung, Jahressonderzahlung, Berufsbildung, Kündigungsschutz, Verdienstsicherung, Arbeitszeit und Rationalisierungsschutz. 
ABB. 3

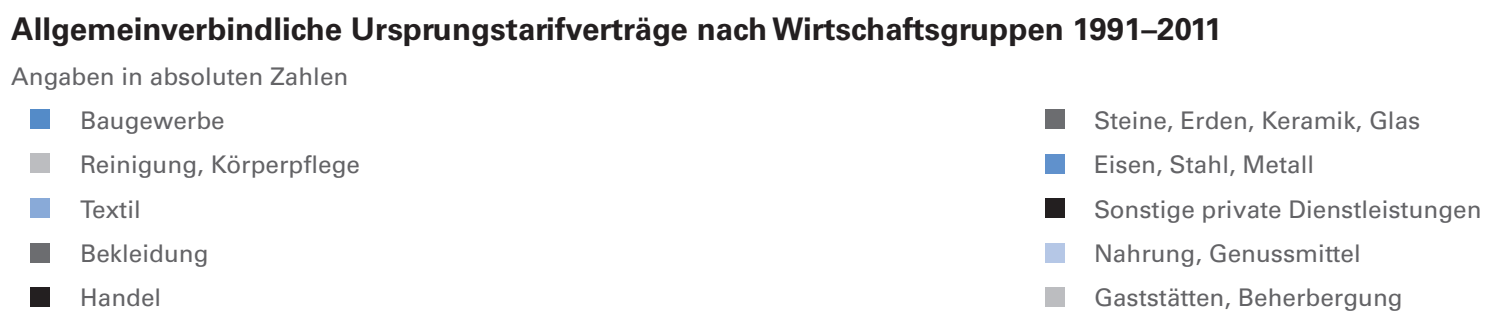

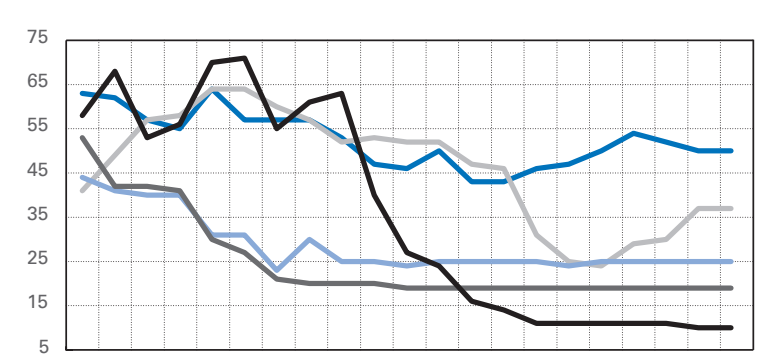

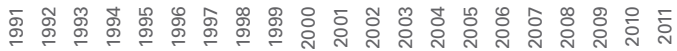

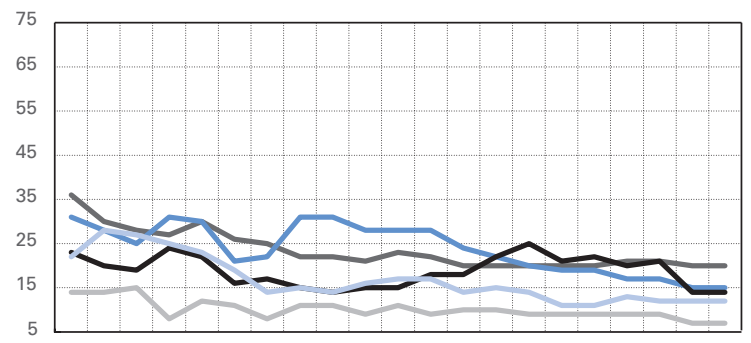

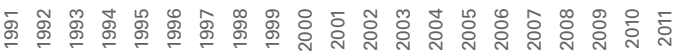

Quelle: BMA-Tarifregister; Berechnungen des Autors.

\section{TABELLE 2}

\section{Allgemeinverbindliche Lohn- und Gehaltstarifverträge (Ursprungstarifverträge) 1949-1985}

Anteil an allen allgemeinverbindlichen Ursprungstarifverträgen in Prozent

\begin{tabular}{c|c|c|c|c|c|c|c|}
\hline $1949 / 50$ & $1954 / 55$ & $1959 / 60$ & $1964 / 65$ & $1969 / 70$ & $1974 / 75$ & $1979 / 80$ & $1984 / 85$ \\
\hline 59,8 & 54,6 & 54,6 & 58,7 & 31,2 & 42,1 & 40,9 & 42,8 \\
\hline
\end{tabular}

Quelle: Wonneberger (1992).
- Friseurhandwerk: Bayern, Hessen, Rheinland-Pfalz, Thüringen,

- Hotel- und Gaststättengewerbe: Niedersachsen,

- Wach- und Sicherheitsgewerbe: Berlin, Brandenburg, Baden-Württemberg, Nordrhein-Westfalen, Niedersachsen, Sachsen, Sachsen-Anhalt, Mecklenburg-Vorpommern.

In drei dieser Bereiche wird jeweils lediglich ein einziges Bundesland erfasst, im Friseurhandwerk sind es vier, im Wach- und Sicherheitsgewerbe sieben Bundesländer. Zum Teil sehen die AVE noch Ausnahmebestimmungen und Begrenzungen auf bestimmte Personengruppen vor. Von einer substanziellen Bedeutung der einkommensbezogenen Allgemeinverbindlicherklärung kann angesichts dieser Sachlage nicht (mehr) die Rede sein. Bemühungen um neue AVE im Bereich von Lohn und Gehalt gestalten sich im Einzelfall sehr schwierig wie Beispiele aus dem Hotel- und Gaststättengewerbe (Flothmann 2011) und dem Einzelhandel (Roth 2012; HDE 2012) zeigen.

\subsection{Allgemeinverbindliche Tarifverträge nach dem AEntG}

Die Zahl der Branchen, für die Mindestlohnbestimmungen auf Basis des Arbeitnehmer-Entsendegesetzes (AEntG) festgelegt wurden, hat sich seit 1996 in mehreren Schritten erweitert. Zunächst waren nur Branchen des Baugewerbes (Bauhauptgewerbe, Dachdeckerhandwerk, Maler- und Lackiererhandwerk, Elektrohandwerk [Montage]) sowie die Seeschifffahrtsassistenz im Geltungsbereich des Gesetzes erfasst; 2007 kamen das Gebäudereini- 
gerhandwerk sowie die Briefdienstleistungen hinzu und mit der Novellierung des Gesetzes im Jahr 2009 wurde der Geltungsbereich um folgende Branchen erweitert: Abfallwirtschaft, Wach- und Sicherheitsgewerbe, Bergbauspezialdienste, industrielle Großwäschereien, Pflegedienste und berufliche Weiterbildung. Zur Diskussion steht ferner die Aufnahme des Bereichs Forstliche Dienstleister in den Geltungsbereich.

Die Höhe der Mindestlöhne auf Basis des AEntG variiert je nach Branche und Region zwischen 7,00 € und 13,40€ (WSI-Tarifarchiv 2012). In fünf Branchen liegen die Mindestlöhne zumindest teilweise unter 8,50 €, und damit unter dem von den Gewerkschaften angestrebten Niveau für einen gesetzlichen Mindestlohn. In acht der zwölf Branchen gibt es unterschiedliche Mindestlöhne in West- und Ostdeutschland, in einer Branche wird ferner die Höhe des Mindestlohns innerhalb der westdeutschen Tarifgebiete differenziert. Lediglich vier Branchen sehen zwei verschiedene Mindestlöhne mit einem höheren Mindestlohn für qualifiziertere Tätigkeiten vor.

\subsection{Erfassung der Beschäftigten durch AVE}

Die Bedeutung der Allgemeinverbindlicherklärung für die Regulierung der Arbeits- und Einkommensbedingungen der Beschäftigten hängt nicht allein von der Zahl der erfassten Tarifverträge und Branchen ab, sondern maßgeblich von der Zahl der erfassten Beschäftigten. Eine Bestandsaufnahme, die auf dem AVE-Verzeichnis vom 1.7.2012 basiert (BMAS 2012), ergibt folgendes Bild:

- Rund 2,4 Mio. Beschäftigte werden von allgemeinverbindlichen Manteltarifverträgen bzw. manteltariflichen Bestimmungen erfasst. Das entspricht rund $13 \%$ der im Geltungsbereich von Tarifverträgen tätigen Beschäftigten.

- Rund 245.000 Beschäftigte werden von allgemeinverbindlichen Einkommenstarifverträgen erfasst. Das entspricht einem Anteil von lediglich 1,3\% der Beschäftigten.

- Rund 3,7 Mio. werden darüber hinaus durch die allgemeinverbindlichen Mindestlohntarifverträge erfasst, die über das Entsendegesetz auch für entsandte Beschäftigte verbindlich sind. ${ }^{(1)}$

Die letztgenannte Zahl überzeichnet allerdings die Bedeutung der Mindestlohntarifverträge, da diese vor allem im unteren Lohn- und Gehaltsbereich eine reale Wirkung entfalten dürften. Belastbare Zahlen über die von den Mindestlöhnen tatsächlich begünstigten Beschäftigten liegen nicht vor.

\subsection{Gründe für den Rückgang der AVE}

Der seit Langem anhaltende Trend zum Rückgang der Allgemeinverbindlicherklärung von Tarifverträgen auf Basis des Tarifvertragsgesetzes lässt sich nach den bisherigen Ausführungen auf verschiedene Faktoren zurückführen:
TABELLE 3

Allgemeinverbindliche Einkommenstarifverträge (Ursprungstarifverträge)*

Angaben in absoluten Zahlen und in Prozent

\begin{tabular}{|c|c|c|c|}
\hline \multirow[b]{2}{*}{ Jahr } & \multicolumn{3}{|c|}{ Einkommenstarifverträge } \\
\hline & Anzahl & $\begin{array}{c}\text { Anteil an allen } \\
\text { allgemeinverbindlichen } \\
\text { Ursprungstarifverträgen } \\
\text { in } \%\end{array}$ & $\begin{array}{c}\text { Anteil an allen gültigen } \\
\text { Ursprungsverbands- } \\
\text { Einkommenstarifverträgen } \\
\text { in } \%\end{array}$ \\
\hline 1991 & 124 & 30 & 5,9 \\
\hline 1992 & 115 & 28 & 5,1 \\
\hline 1993 & 119 & 30 & 5,0 \\
\hline 1994 & 113 & 28 & 4,6 \\
\hline 1995 & 116 & 29 & 4,5 \\
\hline 1996 & 103 & 27 & 4,0 \\
\hline 1997 & 90 & 27 & 3,4 \\
\hline 1998 & 89 & 26 & 3,3 \\
\hline 1999 & 80 & 24 & 2,9 \\
\hline 2000 & 61 & 20 & 2,3 \\
\hline 2001 & 53 & 19 & 1,9 \\
\hline 2002 & 55 & 20 & 2,0 \\
\hline 2003 & 43 & 16 & 1,5 \\
\hline 2004 & 44 & 17 & 1,5 \\
\hline 2005 & 37 & 15 & 1,2 \\
\hline 2006 & 34 & 15 & 1,1 \\
\hline 2007 & 35 & 15 & 1,1 \\
\hline 2008 & 34 & 14 & 1,1 \\
\hline 2009 & 37 & 15 & 1,2 \\
\hline 2010 & 35 & 14 & 1,1 \\
\hline 2011 & 36 & 15 & 1,5 \\
\hline
\end{tabular}

*Allgemeinverbindliche Ursprungstarifverträge jeweils zum Stichtag 1. Januar.

Quelle: BMA-Tarifregister, Berechnungen des Autors.

Zum einen ist in vielen Branchen die Bereitschaft der Arbeitgeberverbände zur Nutzung dieses Instruments geschwunden. Insbesondere der Versuch, Mitglieder in Arbeitgeberverbänden durch den Verzicht auf Tarifbindung zu halten oder neu zu gewinnen, geriet in Widerspruch zur bisherigen AVE-Praxis (vgl. beispielhaft zum Einzelhandel Bispinck et al. 2003). Zum anderen stellt das $50 \%$-Quorum in Zeiten sinkender Tarifbindung oftmals ein ernst zu nehmendes Hindernis dar. Der Beweis für die Erfüllung dieser Voraussetzung durch die beteiligten Tarifparteien ist vielfach nur schwer zu erbringen. Hinzu kommt die deutlich restriktiver gewordene Zustimmungspraxis der BDA bzw. der regionalen Spitzenverbände der Arbeitgeber, was sich auch in der langfristig gestiegenen Ablehnungsquote

(12) Dies schließt auch den Pflegebereich mit 800.000 Beschäftigten ein, wo die Erstreckung über eine Rechtsverordnung erfolgte sowie über die Lohnuntergrenze für Leih-/Zeitarbeitsbeschäftigte. 
widerspiegelt. Schließlich ist in Rechnung zu stellen, dass vor diesem Hintergrund die Bereitschaft der Tarifparteien, AVE-Anträge zu stellen, zurückgegangen ist. Auf diese Weise ist eine sich selbst vorantreibende Spirale in Gang gesetzt worden, die die Zahl der Allgemeinverbindlicherklärungen konstant auf sehr niedrigem Niveau hält. Dies gilt insbesondere für die AVE bei den Einkommenstarifverträgen.

\section{Reformdebatte}

\subsection{Reformanstöße}

Angesichts des seit Langem zu beobachtenden Niedergangs des Instruments der Allgemeinverbindlicherklärung ist es erstaunlich, dass erst in jüngster Zeit eine intensivere Debatte über seine Reform in Gang gekommen ist, obwohl bereits vor einigen Jahren entsprechende Analysen und Reformvorschläge vorgelegt worden sind (vgl. Bispinck et al. 2003; Zachert 2003; Peter et al. 2004; Bispinck/Schulten 2009). Verständlich wird dies allerdings, wenn man die einzelnen Etappen der krisenhaften Entwicklung des Tarifsystems in Deutschland mit ihren unterschiedlichen inhaltlichen Themen und Schwerpunkten in Rechnung stellt. In der ersten Hälfte der 1990er Jahre standen die Lohnpolitik und vor allem die Beschäftigungssicherung im Vordergrund der Tarifpolitik (Bispinck 1995). In Ostdeutschland ging es um die Übertragung der westdeutschen Tarifstrukturen und die schrittweise Anpassung der Tarifstandards an das Westniveau. Die dortigen Konflikte um Härtefall- und Öffnungsklauseln kündigten den Trend zu Dezentralisierung und Verbetrieblichung der Tarifpolitik an. Die Branchentarifbindung von immerhin noch $70 \%$ der Beschäftigten in Westdeutschland gab noch keinen unmittelbaren Anlass zur Sorge.

Dies sollte sich jedoch in den kommenden Jahren rasch ändern. Die Arbeitgeber lernten aus den ostdeutschen Erfahrungen, dass ein „Leben ohne Tarifvertrag“ keineswegs automatisch zu dem von den Gewerkschaften stets angedrohten „Häuserkampf“ führte und eine wachsende Zahl von Arbeitgeberverbänden bot den (potenziellen) Mitgliedern auch eine Verbandsmitgliedschaft ohne Tarifbindung an (Behrens 2011). Der Tarifvertrag als gesellschaftlich selbstverständlicher Regulierungsstandard verlor schrittweise an Rückhalt, die Bindefähigkeit der Arbeitgeberverbände ließ offenkundig nach. Im Übrigen litten auch die Gewerkschaften unter anhaltendem Mitgliederschwund.

Die Machtressourcen der Gewerkschaften schwanden auch aus anderen Gründen: Die seit der scharfen Rezession 1992/93 steigende und bis Mitte der 2000er Jahre anhaltende Massenarbeitslosigkeit veränderte die arbeitsmarktpolitischen Rahmenbedingungen nachhaltig, die zunehmende Einbindung der Wirtschaft in die Weltwirtschaft und der Einzug des Shareholder-Value-Denkens verschärfte die Konkurrenzbedingungen und den Druck auf die betrieblichen Arbeits- und Leistungsstandards. Der vermeintlich verkrustete Arbeitsmarkt und auch das angeblich starre und rigide Tarifvertragssystem wurden zum Objekt der politischen Deregulierungsbegierde. Dies zeigen die Hartz-Reformen ebenso wie auch die Auseinandersetzungen um gesetzliche Öffnungsklauseln für Tarifverträge. In der ersten Hälfte der 2000er Jahre ging es damit weniger um die Wiederbelebung einzelner Regulierungsinstrumente als vielmehr um die Verteidigung grundlegender Prinzipien tarifautonomer Gestaltung der Einkommens- und Arbeitsbedingungen (Bispinck 2003).

Und dennoch wurde durch diese Entwicklungen zugleich auch der Keim für eine neue Reformdebatte zur ReStabilisierung des Tarifsystems gelegt. Die immer klarer zutage tretenden Spaltungstendenzen am Arbeitsmarkt mit einem wachsenden Anteil an prekär Beschäftigten, die zunehmende Einkommenspolarisierung mit einem größer werdenden Niedriglohnsektor und die (teils auch gerade dadurch) abnehmende tarifpolitische Gestaltungsmacht der Gewerkschaften führten zu einer offenen Diskussion innerhalb der Gewerkschaften und im politischen Raum, wie den konkreten inhaltlichen Problemlagen zu begegnen sei und welche Antworten auf der Ebene des Regulierungsinstrumentariums zu geben seien.

\subsection{Mindestlohnregulierung}

Ein erster inhaltlicher Strang dieser Reformdebatte ist mit der Ausweitung des Niedriglohnsektors verknüpft. Die von dem Problem extrem niedriger Arbeitseinkommen auf der Ebene ganzer Branchen betroffene Gewerkschaft NahrungGenuss-Gaststätten (NGG) hatte das Thema bereits in den 1990er Jahren aufgegriffen und die Forderung nach einem branchenübergreifenden gesetzlichen Mindestlohn aufgestellt. Dies lief der traditionellen Orientierung der deutschen Gewerkschaften auf die tarifautonome Gestaltung der Lohnbedingungen zuwider und stieß zunächst auf entsprechende Ablehnung bei den anderen DGB-Gewerkschaften. Eine Ausnahme bildete die Vereinte Dienstleistungsgewerkschaft (ver.di), die in einigen Tarifbereichen mit ähnlichen Problemen konfrontiert war. Erst auf dem DGBKongress 2006 einigten sich die DGB-Gewerkschaften auf eine einheitliche Position zum Thema „Gesetzlicher Mindestlohn“. Für die allgemeine tarifpolitische Reformdebatte war dies von Bedeutung, weil damit von allen DGB-Gewerkschaften zweierlei grundsätzlich anerkannt wurde:

(1) Nicht immer und unter allen Umständen sind Tarifverträge das einzig mögliche und akzeptable Mittel zur Festlegung von Mindesteinkommensbedingungen. Letztlich hängt dies von der organisations- und tarifpolitischen Stärke in den jeweiligen Branchen und Organisationsbereichen ab.

(2) Gesetzliche Regelungen der Lohnfestsetzung bedeuten nicht automatisch einen Eingriff in die Tarifautonomie, sie 
können im Gegenteil zur Stabilisierung des Tarifsystems beitragen und dadurch die Möglichkeiten zur tarifautonomen Gestaltung von Einkommens- und Arbeitsbedingungen verbessern oder gar erst eröffnen.

Die spezifische Entwicklung der deutschen Mindestlohnregulierung hat dann die Aufmerksamkeit erneut auf das Instrument der AVE gelenkt. Zwar war die gewerkschaftliche Kampagne für einen gesetzlichen Mindestlohn insofern ohne Erfolg, als ein bundesweiter, branchenübergreifender Mindestlohn bis dato noch nicht durchgesetzt werden konnte. Doch der politische Druck wurde immerhin so groß, dass sowohl die schwarz-rote Bundesregierung (2005-2009) wie auch die schwarz-gelbe Koalition (seit 2009) in mehreren Schritten branchenbezogene Mindestlöhne einführten. ${ }^{\circledR}$ Wie vorab bereits erläutert, spielt dabei die AVE eine zentrale Rolle. Gemessen an der Zahl der Branchen wie auch der erfassten Beschäftigten sind die allgemeinverbindlichen Mindestlöhne nach dem AEntG inzwischen bei Weitem bedeutender als die klassische AVE für Lohn- und Gehaltstarifverträge. Regulierungspolitisch sind hierbei zwei Punkte von besonderem Interesse: Das Instrument der Rechtsverordnung ( $\$ 7 \mathrm{AEntG}$ ) kann ohne Zustimmung des Tarifausschusses genutzt werden, dessen Blockademöglichkeit entfällt, und das Quorum von $50 \%$ Tarifbindung ist nicht mehr Voraussetzung für eine AVE.

\subsection{Weitere Gestaltungsvorschläge}

In der politisch-strategischen Debatte innerhalb der Gewerkschaften fanden diese Entwicklungen durchaus ihren Niederschlag. So forderte der DGB-Kongress 2006 in einem Initiativantrag „Niedriglohnsektor: Lösungen aus gewerkschaftlicher Sicht“ zur Stärkung der Tarifautonomie sowohl die „Erleichterung der Voraussetzungen zur Erteilung der Allgemeinverbindlicherklärung“ als auch die „Ausweitung des Entsendegesetzes auf alle Wirtschaftsbereiche“ (DGB 2006). Eine vergleichbare Forderung zur Erleichterung der AVE beschloss auch die IG Metall auf ihrem letzten Gewerkschaftstag 2011 in Karlsruhe. Ver.di ging noch ein Stück weiter. Auf dem Gewerkschaftstag 2011 bezeichnete der ver. di-Vorsitzende Frank Bsirske die „grundlegende Reform des jetzigen Verfahrens (als) unumgänglich“. Eine grundlegende Reform müsse so ausgestaltet werden, „dass - ähnlich wie in den Niederlanden - die Mehrheit der Tarifverträge allgemeinverbindlich erklärt werden kann“ (Bsirske 2011). Die Forderung nach einer stärkeren Nutzung von AVE findet auch bei den betrieblichen Interessenvertretungen deutliche Unterstützung. Die WSI-Betriebsrätebefragung 2010 kam zu folgendem Ergebnis: Auf die Frage „Was meinen Sie? Sollten die Tarifverträge in Ihrer Branche für allgemeinverbindlich erklärt werden?" antworteten $83 \%$ der befragten Betriebsräte mit ja. Dabei zeigt sich insgesamt nur eine relativ geringe Differenzierung etwa nach Betriebsgröße, Region (West/Ost) oder Krisenbetroffenheit. Bei den Branchen variiert die Zustimmung immerhin zwischen 77 \% im
Bereich Grundstoffe/Produktionsgüter und 88 \% im Bereich Verkehr/Nachrichten (Bispinck 2010).

Die politische Debatte erhielt neue Anstöße durch Anträge der Bundestagsfraktionen der Oppositionsparteien zur Stärkung des Tarifsystems. Als Erste positionierten sich im Januar 2011 Bündnis 90/Die Grünen gefolgt von der LINKEN im Dezember 2011 und der SPD im Januar 2012. Die Vorschläge bezogen sich u.a. auf folgende Reformaspekte: ${ }^{(4)}$

- Mindestlohn: Alle drei Bundestagsfraktionen sprechen sich für die Einführung eines branchenübergreifenden gesetzlichen Mindestlohns aus.

- Quorum: Die Grünen schlagen eine Absenkung des Quorums von 50 auf $40 \%$ vor, SPD und LINKE befürworten, dass das Quorum entfällt und durch das Kriterium der Repräsentativität des Tarifvertrages nach $₫ 7 \mathrm{AEntG}$ ersetzt wird.

- Tarifausschuss: Er soll nach Auffassung von Grünen und SPD auch mit den jeweiligen Vertretern der Branchen besetzt werden, die eine AVE beantragen. Die Beschlussfassung soll nach dem Mehrheitsprinzip erfolgen. Bei AVE auf Basis von Rechtsverordnungen soll er nur noch Beratungsfunktion haben.

- Inhaltliche Voraussetzung: Neben dem Quorum bzw. der Repräsentativität bleibt es beim Kriterium des öffentlichen Interesses. Die Grünen legen dazu einen Konkretisierungsvorschlag vor.

- AEntG: Alle drei Fraktionen plädieren für Ausweitung des Geltungsbereichs auf alle Branchen. Die LINKE schlägt vor, dass alle Entgelttarifverträge, die das Kriterium der Repräsentativität nach $₫ 7$ AEntG erfüllen, vom BMA für allgemeinverbindlich zu erklären sind.

In der Anhörung des Bundestagsausschusses für Arbeit und Soziales zu diesen Anträgen am 6.2.2012 wurden einerseits die bekannten bzw. erwartbaren Positionen der Verbände bzw. der Wissenschaft vorgetragen, aber es wurden auch neue Argumente entwickelt, die der weiteren Reformdiskussion Impulse geben können (Deutscher Bundestag 2012; Bispinck/Schulten 2012).

Die Bundesvereinigung der Deutschen Arbeitgeberverbände (BDA) lehnte die Vorschläge rundweg ab. Die

(3) Außerdem wurde das Mindestarbeitsbedingungengesetz von 1952 im Jahr 2009 komplett überarbeitet, um die Festsetzung von Arbeitsbedingungen in Branchen ohne bzw. mit sehr geringerTarifbindung zu ermöglichen; es wurde allerdings bis heute in keinem Fall praktisch angewendet.

(4) Zu den Vorschlägen und ihrer rechtlichen und rechtspolitischen Einordnung vgl. im Einzelnen den Beitrag von Däubler in diesem Heft. 
zentrale Argumentation der BDA ging dahin, dass Mindestlöhne und AVE die Tarifautonomie schwächen würden. Dies entspricht der traditionell restriktiven Haltung in Bezug auf die AVE (BDA 2000). Das Präsidium der BDA hatte in einem erneuten Beschluss vom 18.1.2010 Grundsätze zur Allgemeinverbindlichkeit von Tarifverträgen und zur Anwendung des Entsende- und Mindestarbeitsbedingungengesetzes festgelegt (BDA 2010). Darin betont die BDA den Vorrang der Tarifautonomie vor staatlicher Lohnfestsetzung. Die Allgemeinverbindlichkeit von Tarifverträgen müsse die Ausnahme bleiben, es bedürfe immer einer besonderen Rechtfertigung unter den bestehenden engen Voraussetzungen des Tarifvertragsgesetzes. Die Funktion der AVE von Lohn- und Gehaltstarifverträgen bestehe darin, unsozialen Lohn- und Arbeitsbedingungen bei nicht tarifgebundenen Arbeitgebern entgegenzutreten. Nur dann könne ausnahmsweise „wegen besonderem öffentlichen Interesse“ allgemeinverbindlich erklärt werden

Der DGB begrüßte die Absicht der Anträge, Tarifautonomie und Tarifverträge durch eine Ausweitung der AVE zu stärken. Ohne sich abschließend festzulegen, befürwortete er die vorgelegten Einzelmaßnahmen als mögliche Instrumente zur Umsetzung dieser Absichten. Die Anträge beschreiben nach seiner Auffassung deutlich die Notwendigkeit und Anpassung an aktuelle Gegebenheiten. Die Vereinfachung der Allgemeinverbindlicherklärung würde einen wichtigen Beitrag für eine Revitalisierung des Tarifvertragssystems darstellen.

Unter den juristischen Expertisen stach die Stellungnahme von Preis hervor. Er argumentierte auf der Basis eines ausführlichen Gutachtens für die SOKA BAU (vgl. Greiner et al. 2011) insbesondere gegen die Verabsolutierung des 50 \%-Quorums und benannte das Dilemma, dass das Bedürfnis nach staatlichen Einflussmöglichkeiten gerade umso mehr steige, je schwächer sich die Tarifbindung darstelle. „Es ist geradezu grotesk, wenn der sogenannte, Respekt vor der Tarifautonomie' dazu führt, dass in Bereichen, in denen gar keine tarifvertragliche Regulierung existiert, staatliches Tätigwerden unterbleibt. Insofern ist die Wirkungsweise des 50 \%-Quorums schon im Ansatz höchst fragwürdig." Als Alternativen nennt er die moderate oder auch deutlichere Absenkung des Quorums, bevorzugt aber, künftig auf die Fixierung des Quorums zu verzichten und stattdessen auf den offenen, unbestimmten Begriff der „Repräsentativität" auszuweichen, der auch im Recht der europäischen Länder, die eine AVE kennen, umfassend verankert sei. Das Abstellen auf die Repräsentativität habe ein Vorbild in der Tarifvertragsverordnung von 1918, die sich für die Allgemeinverbindlicherklärung mit der „überwiegenden Bedeutung" des Tarifvertrages begnügt und damit durchaus bewährt habe. Denkbar und verfassungsrechtlich legitimierbar sei auch ein völliger Verzicht auf das Quorum.

Ähnlich wie bei der Diskussion um den gesetzlichen Mindestlohn wird auch bei der AVE-Reformdebatte die Aufarbeitung der europäischen Erfahrungen zunehmend wichtig (vgl. WSI Tarifpolitische Tagung 2011). Sie verdeut- licht, dass in einer ganzen Reihe von Ländern mit unterschiedlichen tarifrechtlichen und -politischen Konstellationen die breite Anwendung des AVE-Instruments nachhaltig zur Stabilisierung des Tarifsystems beiträgt. Allerdings wird die AVE in einigen dieser Länder unter dem Druck der derzeitigen Krisen- und Austeritätspolitik aktuell politisch infrage gestellt (vgl. den Beitrag von Schulten in diesem Heft).

\section{Fazit}

20 Jahre rückläufige Tarifbindung, Dezentralisierung und Differenzierung der Tarifverträge haben die Tariflandschaft und auch das konkrete Tarifgeschehen und seine realen Auswirkungen nachhaltig verändert. Die äußere und innere Prägekraft des Flächentarifvertrags als zentrales Instrument der Regulierung von Einkommens- und Arbeitsbedingungen hat stark nachgelassen. Die Reaktionen der Tarifparteien darauf fallen unterschiedlich aus.

Die Arbeitgeberverbandsseite begrüßt die Entwicklung zu stark flexibilisierten und für betriebliche Nachsteuerungen geöffneten Branchentarifverträgen, sie setzt nach wie vor auf deren Ordnungsfunktion und ist von früheren Forderungen nach einer gesetzlichen Öffnungsklausel und einer Veränderung des Günstigkeitsprinzips erst einmal abgerückt. Zugleich setzt sie sich jedoch energisch gegen alle Versuche einer politisch gestützten Re-Stabilisierung des Flächentarifvertrags zur Wehr. Dies gilt insbesondere in Bezug auf die Forderung nach einem einheitlichen gesetzlichen Mindestlohn, aber auch für alle Vorschläge zur Stärkung des Instruments der Allgemeinverbindlicherklärung.

Für die Gewerkschaften stellt sich die Sachlage anders dar. Zwar gehen die meisten Gewerkschaften davon aus, dass es zu einer Strategie der „kontrollierten Dezentralisierung" der Tarifverträge derzeit keine realistische Alternative gibt, aber zugleich wird die Frage nach wirkungsvollen Konzepten gegen das immer weitere Ausfransen des Tarifsystems stärker diskutiert. Ausgangspunkt der Diskussion ist die Erkenntnis, dass es mit dem Einziehen einer gesellschaftlich verbindlichen Lohnuntergrenze durch einen einheitlichen und/oder mehrere branchenbezogene Mindestlöhne allein nicht getan ist. Es geht ihnen darüber hinaus um die Frage, wie dem gesamten Set von Tarifstandards in den einzelnen Branchen wieder zu einer stärkeren Geltung verholfen werden kann. Hier richten sich die Hoffnungen verstärkt auf das Instrument der AVE.

Die weitere Auseinandersetzung um die politisch gestützte Re-Stabilisierung des Tarifsystems bleibt offen. Die Erfahrungen des langjährigen Konflikts um die Einführung eines gesetzlichen Mindestlohnes zeigen: Ein objektiv vorhandener Handlungsspielraum wird nicht auch automatisch politisch genutzt. Entscheidend ist zum einen, in welchem Maße die gesellschaftlichen Akteure politischen Druck für ihre 
Ziele aufbauen können. Dies setzt im konkreten Fall der Reform der AVE sicherlich noch weitere Informations- und Aufklärungsarbeit voraus. Zum anderen hängen die Chancen einer Reform von Tarifvertragsgesetz und Arbeitnehmer-Entsendegesetz von den politischen Rahmenbedingungen genauer: von der politischen Zusammensetzung der nächsten Bundesregierung - ab. Dies haben offenkundig auch die Gewerkschaften im Blick: „Die Hürden für Allgemeinverbindlichkeitserklärungen zu senken, [...] das gehört aktuell zu unseren wichtigsten Forderungen im Vorfeld der Bundestagswahl 2013“" (Bsirske 2011).

\section{LITERATUR}

Artus, I. (2001): Die Krise des deutschen Tarifsystems. Die Erosion des Flächentarifvertrags in Ost und West, Wiesbaden

Bahnmüller, R. (2010): Dezentralisierung derTarifpolitik - Re-Stabilisierung des Tarifsystems?, in: Bispinck, R./Schulten, Th. (Hrsg.): Zukunft derTarifautonomie. 60 Jahre Tarifvertragsgesetz: Bilanz und Ausblick, Hamburg, S. 81-113

Bahnmüller, R./Bispinck, R. (1995): Vom Vorzeige- zum Auslaufmodell? Das deutsche Tarifsystem zwischen kollektiver Regulierung, betrieblicher Flexibilisierung und individuellen Interessen, in: Bispinck, R. (Hrsg.): Tarifpolitik der Zukunft - Was wird aus dem Flächentarifvertrag?, Hamburg, S. 137-172

Behrens, M. (2011): Das Paradox der Arbeitgeberverbände. Von der Schwierigkeit, durchsetzungsstarke Unternehmensinteressen kollektiv zu vertreten, Berlin

Bispinck, R. (1995):Tarifpolitik in der ersten Hälfte der 90er Jahre. Eine zwiespältige Bilanz, in: Ders. (Hrsg.): Tarifpolitik der Zukunft - Was wird aus dem Flächentarifvertrag?, Hamburg, S. 9-27

Bispinck, R. (2003): Das deutsche Tarifsystem in Zeiten der Krise - Streit um Flächentarif, Differenzierung und Mindeststandards, in: WSI-Mitteilungen 56 (7), S. 395-404, http://www.boeckler.de/wsimit_2003_07_bispinck.pdf Bispinck, R. (2010): Niedriglöhne und der Flickenteppich von (unzureichenden) Mindestlöhnen in Deutschland, WSI-Report 04, Düsseldorf

Bispinck, R. (2012): Sozial- und arbeitsmarktpolitische Regulierung durch Tarifvertrag, in: Bispinck, R./Bosch, G./Hofemann, K./Naegele, G. (Hrsg.): Sozialpolitik und Sozialstaat - Festschrift für Gerhard Bäcker, Wiesbaden, S. 201-220 Bispinck, R./Kirsch, J./Schäfer, C. (2003): Mindeststandards für Arbeits- und Einkommensbedingungen und Tarifsystem. Forschungsbericht für das Ministerium für Wirtschaft und Arbeit des Landes NRW, Düsseldorf

Bispinck, R./Schulten, Th. (2008): Aktuelle Mindestlohndebatte: Branchenlösungen oder gesetzlicher Mindestlohn?, in: WSI-Mitteilungen 61 (3),

S. 151-158, http://www.boeckler.de/wsimit_2008_03_bispinck.pdf

Bispinck, R./Schulten, Th. (2009): Re-Stabilisierung des deutschen Flächentarifvertragssystems, in: WSI-Mitteilungen 62 (4), S. 201-209, http://www. boeckler.de/wsimit_2009_04_bispinck_schulten.pdf

Bispinck, R./Schulten, Th. (2012): Stellungnahme zur öffentlichen Anhörung von Sachverständigen vor dem Ausschuss für Arbeit und Soziales des Deutschen Bundestags am 6. Februar 2012, Ausschussdrucksache 17(11)780 vom 31.1

Bsirske, F. (2011): Grundsatzreferat auf dem ver.di-Bundeskongress 2011 in Leipzig, Tagesprotokoll vom 20.9.

Bundesministerium für Arbeit und Soziales (BMAS) (2012): Verzeichnis der für allgemeinverbindlich erklärten Tarifverträge, Stand: 1. Juli

Bundesvereinigung der Deutschen Arbeitgeberverbände (BDA) (2000): Die Allgemeinverbindlicherklärung (AVE) von Tarifverträgen. Orientierung - Grundsätze - Verfahren, Berlin

Bundesvereinigung der Deutschen Arbeitgeberverbände (BDA) (2010): Vorrang derTarifautonomie vor staatlicher Lohnfestsetzung - Grundsätze der Arbeitgeber zur Allgemeinverbindlichkeit von Tarifverträgen und zur Anwendung des Entsende- und Mindestarbeitsbedingungengesetzes, Berlin
Däubler, W. (1997): Die Allgemeinverbindlicherklärung - notwendiges Mittel zum Schutz der Tarifautonomie?, in: Engelen-Kefer, U./Schoden, M./Zachert, U. (Hrsg.): Arbeitsrecht in der Bewährung. Festschrift für Karl Kehrmann, Köln, S. $281-294$

Deutscher Gewerkschaftsbund (DGB) (2006): 18. Ordentlicher DGB-Bundeskongress vom 22.-26.5.2006 in Berlin, Initiativantrag 004 „Niedriglohnsektor: Lösungen aus gewerkschaftlicher Sicht"

Deutscher Bundestag (2012): Ausschuss für Arbeit und Soziales, Materialien zur öffentlichen Anhörung von Sachverständigen in Berlin am 6. Februar 2012, Ausschussdrucksache 17(11)766 vom 2.2

Dombre, R. (2007): Entwicklung des Arbeitnehmer-Entsendegesetzes vom Widerstand im Jahre 1996 bis heute, Manuskript, o.O.

Ellguth, P./Kohaut, S. (2012): Tarifbindung und betriebliche Interessenvertretung: Aktuelle Ergebnisse aus dem IAB-Betriebspanel 2011, in: WSI-Mitteilungen 64 (4), S. 297-305

Flothmann, K. (2011): Hallo Pizza, ist das fair?, in: Die Mitbestimmung 57 (7+8), S. $32-35$

Goldschmidt, H. (1950): Tarifvertragsgesetz und Allgemeinverbindlichkeit von Tarifverträgen, in: Bundesarbeitsblatt, (1), S. 14-17

Greiner, S./Hanau, P./Preis, U. (2011): Die Sicherung der Allgemeinverbindlichkeit bei gemeinsamen Einrichtungen derTarifvertragsparteien, Rechtsgutachten für die SOKA Bau, KöIn/Bochum

Handelsverband Deutschland (HDE) (2012): Einzelhandel gegen gesetzliche Mindestlöhne, Pressemeldung vom 1.6.

Kempen, O. E./Zachert, U. (2006): TVG - Tarifvertragsgesetz. Kommentar für die Praxis, Frankfurt/Main

Kirsch, J. (2003): Die Allgemeinverbindlicherklärung von Tarifverträgen - ein Instrument in der Krise, in: WSI-Mitteilungen 56 (7), S. 405-412, http://www. boeckler.de/wsimit_2003_07_zachert.pdf

Kirsch, J./Bispinck, R. (2002): Allgemeinverbindliche Tarifverträge, Informationen zurTarifpolitik, Düsseldorf

Kreimer-de Fries, J. (1995): Die Allgemeinverbindlicherklärung von Tarifverträgen, in: Bispinck, R. (Hrsg.): Tarifpolitik der Zukunft, S. 203-229

Nautz, J. (1985): Die Durchsetzung derTarifautonomie in Westdeutschland. Das Tarifvertragsgesetz vom 9. April 1949, Frankfurt a. M. et al.

Nautz, J. (1999): Die Entstehung des Tarifvertragsgesetzes, in: WSI-Mitteilungen 52 (7), S. 437-444

Peter, G./Kempen, O. E./Zachert, U. (2004): Die Sicherung tariflicher Mindeststandards. Rechtliche und rechtspolitische Aspekte, Baden-Baden

Reichel, H. (1969): Zwanzig Jahre Allgemeinverbindlicherklärung von Tarifverträgen, in: Bundesarbeitsblatt (5), S. 359-372

Roth, E. (2012): Einzelhandel: Mindestlohn ade, in: Frankfurter Rundschau vom 30.5., http://www.fr-online.de/wirtschaft/einzelhandel-mindestlohnade, $1472780,16146920$. html

Tijdens, K./v. Klaveren, M. (2009): Measuring IR in the EU: concepts and outcomes using Wagelndicator web-survey data, Amsterdam

Wiedemann, H. (2007): Tarifvertragsgesetz, München

Wonneberger, W. (1992): Die Funktionen der Allgemeinverbindlicherklärung von Tarifverträgen, Heidelberg

WSI-Tarifarchiv (2012): Tarifliche Mindestlöhne nach dem Arbeitnehmer-Entsendegesetz und dem Arbeitnehmer-Überlassungsgesetz, http://www.boeckler. de/pdf/ta_mindestloehne_aentg.pdf

WSI Tarifpolitische Tagung (2011): Stabilisierung des Flächentarifvertrages Reform der Allgemeinverbindlicherklärung, Tagung am 27. und 28.9.2012, Dokumentation derTagungsbeiträge unter: http://www.boeckler.de/28733_36062. htm

Zachert, U. (2003): Reformperspektiven der Allgemeinverbindlicherklärung aus arbeitsrechtlicher Sicht, in: WSI-Mitteilungen 56 (7), S. 413-419, http:// www.boeckler.de/wsimit_2003_07_zachert.pdf

\section{AUTOR}

REINHARD BISPINCK, Dr., ist Wissenschaftler im Wirtschafts- und Sozialwissenschaftlichen Institut (WSI) und Leiter des WSI-Tarifarchivs in der HansBöckler-Stiftung.

reinhard-bispinck@boeckler.de 